\title{
Effects of Laparoscopic Sleeve Gastrectomy on Bone Mineral Density and Bone Metabolism in Chinese Patients with Obesity
}

This article was published in the following Dove Press journal: Diabetes, Metabolic Syndrome and Obesity: Targets and Therapy

\author{
Xi Chen' \\ Chunlan Zhang' \\ Jingping $\mathrm{Li}^{2}$ \\ Wei Liu ${ }^{2}$ \\ Jingjing Zhang (D)' \\ Zhiguang Zhou' \\ 'National Clinical Research Center for \\ Metabolic Diseases, Metabolic Syndrome \\ Research Center, Key Laboratory of \\ Diabetes Immunology (Central South \\ University), Ministry of Education, \\ Department of Metabolism and \\ Endocrinology, The Second Xiangya \\ Hospital of Central South University, \\ Changsha 4I00II, Hunan, People's \\ Republic of China; ${ }^{2}$ Department of \\ Metabolic Surgery, Department of \\ Biliopancreatic Surgery, The Second \\ Xiangya Hospital, Central South \\ University, Changsha 4I00II, Hunan, \\ People's Republic of China
}

Objective: Laparoscopic sleeve gastrectomy (LSG) is one of the most effective therapies to treat obesity. Due to a lack of longitudinal research, we explored the changes in BMD and bone metabolism in Chinese patients with obesity before and after LSG.

Materials and Methods: A total of 49 obese Chinese patients with obesity were recruited from the Second Xiangya Hospital of Central South University. All patients underwent LSG, and the metabolic indicators were evaluated, including the serum osteocalcin (OC), totalterminal propeptide of type I procollagen (TPINP) and carboxy terminal telopeptide of collagen type I (CTX) levels at baseline and 2, 6 and 12 months postoperatively. Dual energy X-ray absorptiometry (DEXA) was used to measure body composition and BMD before and 12 months after LSG.

Results: The body mass index (BMI) significantly decreased at 12 months postoperatively, and no patients developed osteoporosis. The BMD of femoral neck and total hip significantly decreased from the baseline to 12 months postoperatively, while the BMD of lumbar spine did not change significantly. The OC, TPINP and CTX levels significantly increased at 12 months postoperatively. The excess BMI loss (EBMIL) was positively related while the reduction in visceral adipose tissue (VAT) mass was negatively related to the decreases in BMD of the femoral neck and total hip at 12 months postoperatively. The increase in OC was negatively correlated with the decrease in BMD of the femoral neck, while the increase in TPINP was positively correlated with the decrease in BMD of total hip.

Conclusion: Based on the 12-month follow-up results, the BMD of the femoral neck and total hip was decreased in Chinese patients with obesity after LSG, while bone remodelling was active. This finding suggested that weight loss, a decrease in the VAT mass and an increase in bone remodelling may be correlated with a reduction in BMD.

Keywords: laparoscopic sleeve gastrectomy, obesity, bone mineral density, bone metabolism markers

\section{Introduction}

Obesity is an important public health concern worldwide, and the global prevalence of obesity in adults has been increasing in recent decades. ${ }^{1}$ Metabolic surgeries, such as laparoscopic sleeve gastrectomy (LSG) and Roux-en-Y gastric bypass (RYGB), are effective interventions that can lead to marked weight loss and improved glucose control $^{2}$ and have been considered one of the treatments for obesity. ${ }^{3}$ The LSG procedure has gained popularity in the past decade because it is easy to perform and is associated with a low incidence of serious complications. ${ }^{4}$ The effects of LSG on BMD in the Chinese population are not yet clear.
Correspondence: Jingjing Zhang Metabolic Diseases, Metabolic Syndrome Research Center, Key Laboratory of Diabetes Immunology (Central South University), Ministry of Education, Department of Metabolism and Endocrinology, The Second Xiangya Hospital of Central South University, Changsha 4I00II, Hunan, People's Republic of China

Email Doctorzhangji@csu.edu.cn 
Many factors cause a decrease in BMD and increase the risk of osteoporosis and fractures, including a low body mass index (BMI), ${ }^{5}$ insufficient protein intake, and calcium and vitamin D deficiencies. ${ }^{6}$ Research results on the relationship between obesity and BMD are not consistent. It has been demonstrated that women with obesity have higher volumetric BMD in the lumbar spine and that both men and women with obesity have higher volumetric BMD in the distal radius and distal tibia than normal weight individuals. $^{7}$ In addition, a positive association between BMI and BMD has been identified. ${ }^{8}$ On the other hand, researchers have also found that a high BMI can interfere with bone metabolism, which may cause bone loss and an increased risk of fractures. ${ }^{9,10}$ It has also been suggested that adipose tissue mass may have a dual effect on BMD, and too much or too little fat mass can have adverse effects on the bone. ${ }^{11}$ Overweight and underweight are associated with an increased incidence of fractures at various sites. ${ }^{12}$ The above research results suggest the importance of maintaining a normal BMI for the prevention of osteoporosis.

The existing studies on the effects of metabolic surgery on bone have mainly involved RYGB. ${ }^{13}$ The results on the changes in BMD after LSG are inconsistent. ${ }^{14-18}$ It has been shown that femoral neck BMD decreases, spine BMD increases, and total body BMD remains unchanged in men with obesity after LSG, ${ }^{14}$ while another study has shown that BMD increases during the first and second years after LSG. ${ }^{15}$ In addition, researchers have found that BMD decreases after LSG, ${ }^{16-18}$ and the effect of LSG on BMD differs between genders, decreasing significantly in females while remaining unchanged in males. ${ }^{18}$ The effects on BMD and bone metabolism in the Chinese population remain unknown. The purposes of our study were to investigate the effect of LSG on the BMD and bone metabolism of Chinese people with obesity and to explore clinical factors that may be related to changes in BMD.

\section{Materials and Methods}

\section{Participants}

In this observational cohort study, 49 subjects with obesity were recruited from the Second Xiangya Hospital of Central South University (CSU) (Changsha, Hunan, China). LSG was performed on all patients with obesity who met the surgical criteria. ${ }^{3}$ The exclusion criteria for all the participants were diseases or medications known to affect bone metabolism (hyperthyroidism, hyperparathyroidism, Cushing, chronic kidney disease, glucocorticoids, osteoporosis therapy, hormone replacement therapy, etc.); type 1 diabetes mellitus; secondary diabetes; inflammation, infectious diseases, or other autoimmune disorders; pregnancy; and malignant diseases. This study protocol was conducted in accordance with the Declaration of Helsinki and was approved by the ethics committee of the Second Xiangya Hospital of CSU. All participants provided informed consent for the study programme.

\section{Study Design and Laboratory Analysis}

Subjects with obesity underwent assessments (after $10 \mathrm{~h}$ of fasting overnight) within 1 week before, 2 months after, 6 months after and 12 months after surgery. Study physicians recorded the body height, weight and waist circumference before and 2, 6, and 12 months after the therapy. Body mass index (BMI) was calculated as the body weight $(\mathrm{kg}) /$ height $(\mathrm{m})^{2}$, and excess BMI loss (EBMIL) (\%) was calculated as $\left(\mathrm{BMI}_{\text {baseline }}-\mathrm{BMI}_{\text {follow-up }}\right) /\left(\mathrm{BMI}_{\text {baseline }}-24\right)$. It has been recommended that serum total-terminal propeptide of type I procollagen (TPINP) and osteocalcin (OC) levels are used as reference bone formation markers and that serum carboxy terminal telopeptide of collagen type I (CTX) is used as the reference bone resorption marker. ${ }^{19}$ Fasting venous blood samples were tested for albumin, serum calcium, serum phosphorus, intact parathyroid hormone (iPTH), 25-OH-vitamin D, serum OC, serum TPINP, serum CTX, fasting plasma glucose (FPG), fating C-peptide (FCP) and haemoglobin $\mathrm{A}_{1 \mathrm{c}}\left(\mathrm{HbA}_{1 \mathrm{c}}\right)$. A dualenergy X-ray absorptiometry (DEXA) body fat composition analyser and bone density scanner (Discovery, HOLOGIC, Bedford, MA) were used to measure BMD in the lumbar spine, femoral neck and total hip, fat mass, lean mass and visceral adipose tissue (VAT) mass. All scans were performed with the same device for each individual. The definition of osteoporosis assessed by DEXA was a T-score of $\leq-2.5$ and a Z-score of $\leq-2$, and that of osteopenia was a T-score of $\leq-1$ and $>-2.5 .^{20}$ The definition of the clinically significant reduction in BMD $\left(>0.03 \mathrm{~g} / \mathrm{cm}^{2}\right)$ used the smallest detectable difference. ${ }^{21}$

\section{Definitions of Vitamin D Deficiency and Supplementation}

Vitamin D deficiency was defined as a 25-OH-vitamin D of $<20 \mathrm{ng} / \mathrm{mL}$, and vitamin D insufficiency as $25-\mathrm{OH}-$ vitamin D of 21-29 ng/mL. ${ }^{22}$ Most (39/49) subjects were diagnosed with vitamin D deficiency before LSG in our 
study. All the participants were instructed to take vitamin D $800 \mathrm{IU}$ and Ca $300 \mathrm{mg}$, as the levels of 25-OH-vitamin D were $<30 \mathrm{ng} / \mathrm{mL}$.

\section{Surgical Procedure}

All LSG surgeries were performed in the Department of Metabolic Surgery at the Second Xiangya Hospital of CSU.

\section{Statistical Analysis}

Data are presented as the mean \pm SD for normally distributed parameters or as the median (25th-75th percentile) for nonnormally distributed parameters. To compare the changes from baseline to the postsurgical time points within groups, repeated measures ANOVA with the Bonferroni post hoc test was performed. A multivariable linear regression analysis was performed to identify the clinical factors associated with changes in BMD. For the statistical analysis, SPSS version 20.0 (IBM Corporation, Chicago, IL) and GraphPad Prism 5 (GraphPad Software, San Diego, CA) software were used. A two-tailed $P<0.05$ was considered statistically significant.

\section{Results}

\section{The Metabolic Indicators Significantly} Improved After LSG, While the Levels of 25-OH-Vitamin D Were Deficient Before

\section{and After LSG}

The anthropometric and laboratory data are listed in Table 1. At baseline, the age of the patients with obesity was $29.3 \pm$ 1.3 years. Compared with baseline, the BMI significantly decreased in the patients with obesity at 12 months after LSG $\left(27.2 \pm 0.5\right.$ vs $\left.40.0 \pm 0.8 \mathrm{~kg} / \mathrm{m}^{2}, P=0.000\right)$. The EBMIL was $81.6 \pm 2.9 \%$ at 12 months after LSG. The waist circumference, $\mathrm{FPG}, \mathrm{FCP}, \mathrm{HbA}_{1 \mathrm{c}}$, fat mass, lean mass and VAT mass all significantly decreased in the patients with obesity from the baseline to 12 months after LSG $(P<0.05)$. The albumin and serum calcium levels increased at 12 months after LSG ( $P=0.000$ and 0.004 , respectively). The 25-OH-vitamin D, iPTH and serum phosphorus levels were not significantly different from the baseline to 12 months after LSG $(P>0.05)$. The levels of 25-OH-vitamin D before and after surgery were both deficient, while the levels of serum calcium, phosphorus and iPTH were in the normal ranges. We used each patient's last follow-up visit to determine the use of vitamin D supplements. A total of 22 (44.9\%) patients were still using vitamin D supplements at 12 months, while $27(55.1 \%)$ patients never used them during the 12 months. There was no significant difference in the levels of 25-OH-vitamin D between the patients with and without vitamin D supplementation.

\section{Serum Bone Metabolism Markers Increased the in Patients with Obesity After LSG}

As shown in Figure 1A and B, the levels of the bone formation markers, ${ }^{19}$ serum OC $(P=0.000, P=0.000$, $P=0.000$, respectively $)$ and TPINP $(P=0.001, P=0.000$, $P=0.000$, respectively), significantly increased at 2 months, 6 months and 12 months after LSG compared with baseline. Moreover, the median percentage increases in serum OC and TPINP were $+100.0 \%$ and $+45.1 \%$, respectively, at 12 months after LSG. As shown in Figure $1 \mathrm{C}$, the bone resorption marker ${ }^{19}$ serum CTX also significantly increased at 2 months, 6 months and 12 months after LSG compared with baseline $(P=0.000$, $P=0.000$ and 0.000 , respectively). The median percentage increases in serum CTX were $+85.9 \%,+79.3 \%$ and $+52.8 \%$ at 2 months, 6 months and 12 months after LSG, respectively. As shown in Supplementary Table 1, serum OC, TPINP, and CTX increased at 12 months after LSG in both the female and male subjects with obesity (all $P<0.05$ ). As shown in Supplementary Table 2, serum OC, TPINP, CTX increased at 12 months after LSG in the subjects with obesity with T2DM and without T2DM (all $P<0.05)$.

\section{BMD of the Femoral Neck and Total Hip Decreased in the Patients with Obesity After LSG}

As shown in Table 2, there were 2 patients (4.1\%) with osteopenia at baseline, while there were 8 patients (16.3\%) with osteopenia and no patients with osteoporosis or fractures at 12 months after LSG. A clinically significant reduction in BMD $\left(>0.03 \mathrm{~g} / \mathrm{cm}^{2}\right)$ at 1 or more sites was observed in 43 patients (87.8\%) at 12 months after LSG. As shown in Figure 2A, the BMD of femoral neck and total hip significantly decreased at 12 months after LSG ( $P=0.000, P=0.000$, respectively), while the BMD of lumbar spine was not significantly changed at 12 months after LSG. In Figure 2B, the Z-scores of femoral neck, total hip and lumbar spine decreased at 12 months after LSG $(P=0.000, P=0.000, P=0.000$, respectively). As shown in Figure $2 \mathrm{C}$, the T-scores of the femoral neck, total 
Table I Anthropometric and Laboratory Data of the Subjects with Obesity

\begin{tabular}{|c|c|c|c|c|}
\hline & Baseline & 2 Months & 6 Months & 12 Months \\
\hline Age (years) & $28.0(23.5-35.0)$ & & & \\
\hline Gender (female/male) & $20 / 29$ & & & \\
\hline Hormone status (menopause/not menopause) & $0 / 20$ & & & \\
\hline Smoking status (non-smokers/smokers) & $45 / 4$ & & & \\
\hline T2DM (with/without) & $32 / 17$ & $0 / 49$ & $0 / 49$ & $0 / 49$ \\
\hline $\mathrm{BMI}\left(\mathrm{kg} / \mathrm{m}^{2}\right)$ & $40.0 \pm 5.4$ & $32.8 \pm 4.1^{* * * *}$ & $29.3 \pm 3.2 * * *$ & $27.2 \pm 3.3 * * *$ \\
\hline EBMIL (\%) & 1 & $48.8 \pm 19.4$ & $69.8 \pm 18.3$ & $81.6 \pm 20.1$ \\
\hline Waist (cm) & $121.8 \pm 13.0$ & $106.8 \pm 10.5 * * *$ & $97.2 \pm 9.3 * * *$ & $91.0 \pm 15.7 * * *$ \\
\hline FPG (mmol/L) & $5.8(4.6-7.9)$ & $4.7(4.2-5.2)^{* * *}$ & $4.5(4.2-4.8)^{* * *}$ & $4.4(4.0-4.8)^{* * *}$ \\
\hline FCP (pmol/L) & $1063.2(8 \mid 3.6-1375.3)$ & $756.4(532.6-980.5)^{* * *}$ & $612.5(460.0-806.5)^{* * * *}$ & $500.6(372.3-647.5)^{* * * *}$ \\
\hline $\mathrm{HbA} \mathrm{A}_{\mathrm{IC}}(\%)$ & $7.3 \pm 2.0$ & $5.4 \pm 0.7 * * *$ & $5.3 \pm 0.5 * * *$ & $5.3 \pm 0.5^{* * *}$ \\
\hline Albumin $(\mathrm{g} / \mathrm{L})$ & $40.8 \pm 3.0$ & $44.1 \pm 3.5^{* * * *}$ & $44.4 \pm 3.0 * * *$ & $44.8 \pm 2.9 * * *$ \\
\hline $25-\mathrm{OH}$-vitamin $\mathrm{D}(\mathrm{ng} / \mathrm{mL})$ & $14.3 \pm 5.2$ & $16.8 \pm 7.8^{*}$ & $15.7 \pm 7.9$ & $16.0 \pm 5.3$ \\
\hline Serum calcium (mmol/L) & $2.22 \pm 0.11$ & 1 & $2.37 \pm 0.20 * * *$ & $2.32 \pm 0.20 * *$ \\
\hline Serum phosphorus (mmol/L) & $1.25 \pm 0.21$ & 1 & $1.32 \pm 0.16^{*}$ & $1.30 \pm 0.17$ \\
\hline iPTH (pmol/L) & $4.36 \pm 2.08$ & 1 & $4.04 \pm 1.27$ & $4.19 \pm 1.85$ \\
\hline Fat mass $(\mathrm{g})$ & $45409.6 \pm 11764.2$ & 1 & $29117.6 \pm 8136.5^{* * * *}$ & $25316.8 \pm 7775.0 * * *$ \\
\hline Lean mass $(\mathrm{g})$ & $60759.4 \pm 11862.4$ & 1 & $48744.8 \pm 9118.4 * * *$ & $47991.8 \pm 8878.8 * * *$ \\
\hline VAT mass (g) & $1100.2 \pm 239.1$ & 1 & $643.4 \pm 202.3^{* * * *}$ & $571.8 \pm 176.9^{* * *}$ \\
\hline$O C(n g / m L)$ & $14.9 \pm 9.1$ & $21.7 \pm 8.2 * * *$ & $25.1 \pm 8.0 * * *$ & $27.6 \pm 11.1$ **** \\
\hline TPINP (ng/mL) & $73.53 \pm 35.05$ & $85.04 \pm 31.18^{* *}$ & $101.08 \pm 32.86 * * *$ & $98.31 \pm 40.02 * * *$ \\
\hline CTX (pg/mL) & $534.4 \pm 219.9$ & $957.4 \pm 414.7^{* * *}$ & $922.8 \pm 304.1 * * *$ & $831.4 \pm 300.8^{* * *}$ \\
\hline
\end{tabular}

Notes: Data are presented as the mean \pm SD or the median $(25$ th -75 th percentile). $* P<0.05$ compared with baseline, $* * P<0.01$ compared with baseline, $* * * P<0.001$ compared with baseline.

Abbreviations: BMI, body mass index; CTX, carboxy terminal telopeptide of collagen type I; EBMIL, excess BMI loss; FCP, fating C-peptide; FPG, fasting plasma glucose; $\mathrm{HbA}_{I c}$, haemoglobin $\mathrm{A}_{\mathrm{Ic}}$; iPTH, intact parathyroid hormone; OC, osteocalcin; T2DM, type 2 diabetes mellitus; TPINP, total-terminal propeptide of type I procollagen; VAT, visceral adipose tissue.

hip and lumbar spine also decreased at 12 months after LSG $(P=0.000, P=0.000, P=0.000$, respectively). As shown in Supplementary Table 1, the BMD of femoral neck and total hip in the female subjects with obesity were significantly lower than in the male subjects with obesity at both baseline and 12 months after LSG (all $P<0.05$ ). As shown in Supplementary Table 1, compared with baseline, the BMD of femoral neck and total hip significantly decreased at 12 months after LSG in both the female and male subjects with obesity (all $P<0.05$ ), while the BMD of lumbar spine was not significantly changed at 12 months after LSG (all $P>0.05$ ). As shown in Supplementary Table 2, compared with baseline, the BMD of femoral neck and total hip were significantly decreased at 12 months after LSG in the subjects with obesity with T2DM and without T2DM (all $P<0.05$ ),
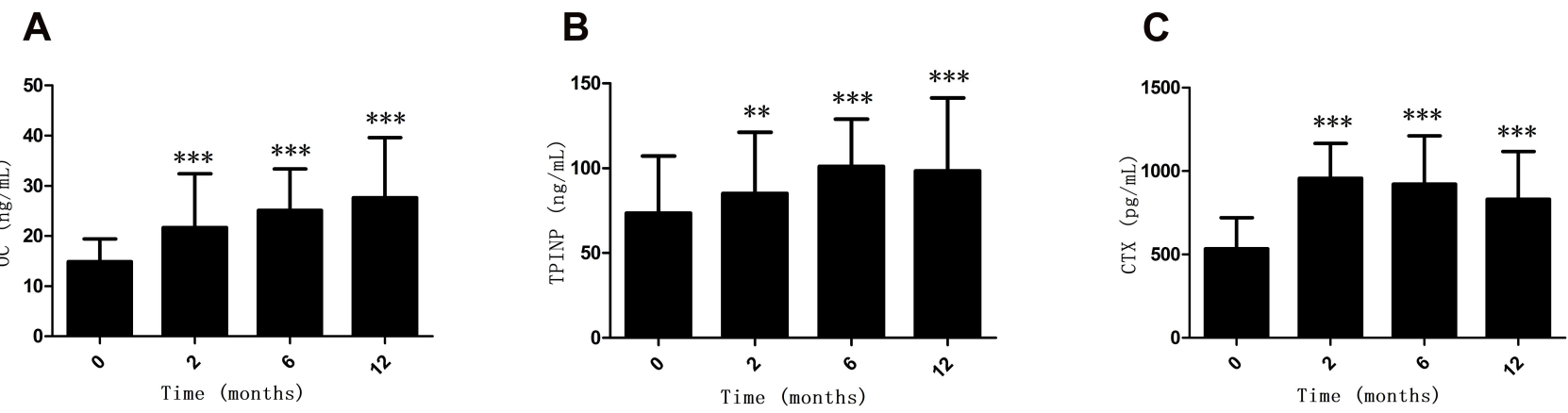

Figure I Alterations (mean $\pm S D$ ) in the levels of OC $(\mathbf{A})$, TPINP $(\mathbf{B})$ and CTX $(\mathbf{C})$ in patients with obesity during the 12 months after LSG. $* * P<0.0$ I, $* * * P<0.00$ I compared with the baseline.

Abbreviations: CTX, carboxy terminal telopeptide of collagen type I; OC, osteocalcin; TPINP, total-terminal propeptide of type I procollagen. 
Table 2 Variations in Bone Mineral Density of Subjects with Obesity

\begin{tabular}{|c|c|c|}
\hline & Baseline & 12 Months \\
\hline $\begin{array}{l}\text { Bone status (normal/osteopenia/ } \\
\text { osteoporosis) } \\
\text { Sites of BMD reduction }>0.03 \mathrm{~g} / \mathrm{cm}^{2} \\
(0 / 1 / 2 / 3)\end{array}$ & $\begin{array}{l}47 / 2 / 0 \\
1\end{array}$ & $\begin{array}{l}4 I / 8 / 0 \\
6 / I I / 2 I / I I\end{array}$ \\
\hline $\begin{array}{l}\text { Total body } \\
\text { BMD }\left(\mathrm{g} / \mathrm{cm}^{2}\right) \\
\text { T-score } \\
\text { Z-score }\end{array}$ & $\begin{array}{l}1.22 \pm 0.15 \\
0.50 \pm 1.21 \\
0.52 \pm 1.21\end{array}$ & $\begin{array}{l}1.21 \pm 0.12 \\
0.53 \pm 1.20 \\
0.52 \pm 1.17\end{array}$ \\
\hline $\begin{array}{l}\text { Femoral neck } \\
\text { BMD }\left(\mathrm{g} / \mathrm{cm}^{2}\right) \\
\text { T-score } \\
\text { Z-score }\end{array}$ & $\begin{array}{l}0.97 \pm 0.15 \\
1.29 \pm 1.46 \\
1.56 \pm 1.59\end{array}$ & $\begin{array}{l}0.91 \pm 0.14^{* * *} \\
0.57 \pm 1.38^{* * *} \\
0.76 \pm 1.41 * * *\end{array}$ \\
\hline $\begin{array}{l}\text { Total hip } \\
\text { BMD }\left(\mathrm{g} / \mathrm{cm}^{2}\right) \\
\text { T-score } \\
\text { Z-score }\end{array}$ & $\begin{array}{l}1.14 \pm 0.12 \\
2.25 \pm 2.52 \\
2.23 \pm 1.24\end{array}$ & $\begin{array}{l}1.02 \pm 0.13^{* * *} \\
0.65 \pm 1.14^{* * *} \\
0.84 \pm 1.26^{* * *}\end{array}$ \\
\hline $\begin{array}{l}\text { Lumbar spine } \\
\text { BMD }\left(\mathrm{g} / \mathrm{cm}^{2}\right) \\
\text { T-score } \\
\text { Z-score }\end{array}$ & $\begin{array}{l}1.08 \pm 0.13 \\
0.76 \pm 1.16 \\
1.25 \pm 1.29\end{array}$ & $\begin{array}{l}\mathrm{I} .07 \pm 0.13 \\
0.50 \pm \mathrm{I} .24 * * \\
0.75 \pm 1.36 * * *\end{array}$ \\
\hline
\end{tabular}

Notes: Data are presented as the mean \pm SD. $* * P<0.01$, $* * * P<0.001$ compared with baseline.

Abbreviation: BMD, bone mineral density.

while the BMD of lumbar spine was not significantly changed at 12 months after LSG (all $P>0.05$ ).

\section{The EBMIL and the Decrease in the VAT Mass Were Correlated with the Decrease in BMD of the Femoral Neck and Total Hip After LSG}

Multiple linear regression analyses were performed with the changes in BMD as dependent variables and the changes in clinical indicators as independent variables from the baseline to 12 months after LSG. As shown in Table 3, the reduction in VAT mass and the increase in OC during the 12 months after LSG were found to be significantly inversely associated with the decrease in BMD of the femoral neck $(P=0.015, P=0.012$, respectively), while the EBMIL was positively related to the decrease in BMD of the femoral neck $(P=0.008)$. The decrease in VAT mass during 12 months after LSG was also found to be significantly negatively related to the decrease in BMD of the total hip $(P=0.026)$, while the EBMIL and the increase in CTX were positively related to the decrease in BMD of the femoral neck $(P=0.022, P=0.045$, respectively).

\section{Discussion}

As one of the standard treatments for patients with obesity, metabolic surgery has attracted increasing attention due to its safety. The BMD changes after metabolic surgery reported in the current literature mainly occur following RYGB. ${ }^{13}$ Our research demonstrated that the BMD of the femoral neck and total hip decrease in patients with obesity after LSG, while the markers of bone formation and resorption all increase after LSG. Since all the subjects underwent LSG, our research added the evidence of the effects of LSG on BMD and bone metabolism in Chinese patients with obesity.

In contrast to previous studies showing that LSG has no effect on BMD in Chileans ${ }^{23}$ or increases the BMD in Czechs and Spanish, ${ }^{14,15}$ our study showed that the BMD of the femoral neck and total hip in the Chinese population with obesity decrease after LSG. As the changes in the BMD percentage may only partially reflect the physiological changes, we chose to compare the absolute values of BMD before and after surgery. In our study, regardless of gender, the BMD of the femoral neck and total hip both decreased in Chinese patients with obesity after LSG (Figure 2A and Supplementary Table 1). This was different from previous reports in Chinese populations which showed that the effect of LSG on BMD differed between genders, decreasing significantly in females while remaining unchanged in males. ${ }^{18}$ The number of patients diagnosed with osteopenia increased at 12 months, and no patients were diagnosed with osteoporosis or fractures (Table 2). Moreover, clinically significant reductions in BMD $\left(>0.03 \mathrm{~g} / \mathrm{cm}^{2}\right)$ at 1 or more sites were observed in 43 patients (87.8\%) at 12 months after LSG (Table 2). The average age of the patients with obesity was $29.3 \pm 1.3$, and we found that the Z-scores of the femoral neck, total hip and lumbar spine decreased after LSG (Figure 2B). The above evidence suggests that the BMD of the Chinese population with obesity in our study decreased after LSG. A lower BMD is linked to a higher risk of fracture, ${ }^{5}$ and a significant association between metabolic surgery and fracture has been reported. ${ }^{24-26}$ It is worth noting that RYGB but not LSG increased the risk of major osteoporotic fractures in a French population aged 40 to 65 years. ${ }^{27}$ The decline in BMD caused concern because we could not rule out the idea that weight loss after surgery caused 
A

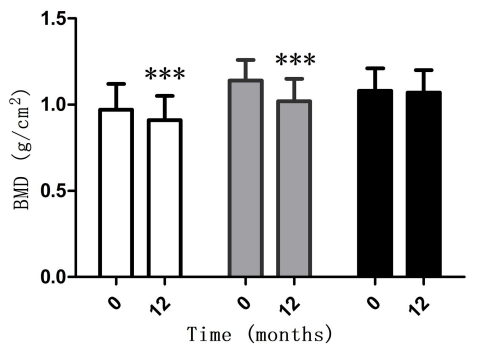

B

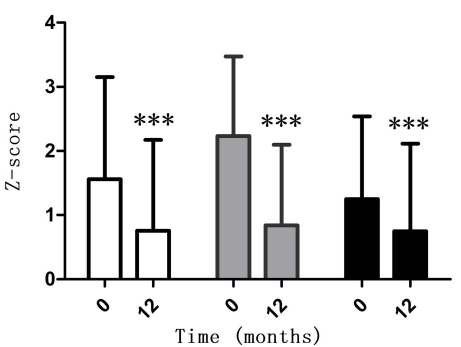

C

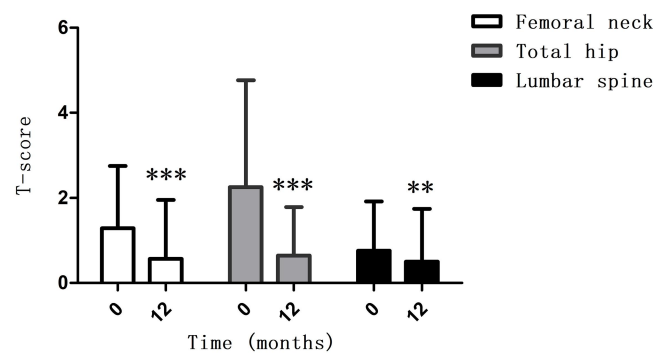

Figure 2 Alterations (mean \pm SD) in the BMD (A) Z-score $(\mathbf{B})$ and T-score $(\mathbf{C})$ of the femoral neck, total hip and lumbar spine during the 12 months after LSG. **P $<0.01$, $* * * P<0.001$ compared with the baseline.

Abbreviation: BMD, bone mineral density.

skeletal fragility. Although no cases of osteoporosis or fracture were observed in our study, a longer follow-up period for observations is needed to determine the risk of long-term postoperative osteoporosis and fracture.

In our study, the levels of all the markers of bone formation and resorption increased after LSG (Table 1 and Figure 1). With a decrease in BMD, elevated serum concentrations of bone formation and resorption markers after RYGB and LSG compared with before surgery have been reported. ${ }^{28,29}$ Therefore, we believe that bone remodelling actively occurs in patients with obesity after both RYGB and LSG, as bone formation and bone resorption co-exist. Interestingly, we found that the rate of increase in CTX, which was a marker of bone resorption, peaked at 2 months after LSG and then decreased gradually (Figure 1C). This result may indicate that the bone resorption rate decreases gradually after 2 months, but the reason remains unclear. Although researchers found that the observed reduction in BMD possibly reflects an

Table 3 Multiple Linear Regression Analysis of the Changes in BMD and Clinical Data During the 12 Months After LSG

\begin{tabular}{|l|l|l|l|l|}
\hline $\begin{array}{l}\text { Dependent } \\
\text { Variables }\end{array}$ & $\begin{array}{l}\text { Independent } \\
\text { Variables }\end{array}$ & $\boldsymbol{\beta}$ & $\mathbf{t}$ & $\boldsymbol{P}$ \\
\hline $\begin{array}{l}\Delta \text { BMD in } \\
\text { femoral neck }\end{array}$ & EBMIL & 0.497 & 2.821 & $\mathbf{0 . 0 0 8}$ \\
\cline { 2 - 5 } & $\Delta$ VAT mass & -0.471 & -2.564 & $\mathbf{0 . 0 1 5}$ \\
\cline { 2 - 5 } & $\Delta$ OC & -0.402 & -2.666 & $\mathbf{0 . 0 1 2}$ \\
\hline \multirow{2}{*}{$\begin{array}{l}\Delta \text { BMD in total } \\
\text { hip }\end{array}$} & EBMIL & 0.375 & 2.398 & $\mathbf{0 . 0 2 2}$ \\
\cline { 2 - 5 } & $\Delta$ VAT mass & -0.370 & -2.069 & $\mathbf{0 . 0 3 8}$ \\
\cline { 2 - 5 } & $\Delta$ CTX & 0.351 & 2.523 & $\mathbf{0 . 0 2 6}$ \\
\hline
\end{tabular}

Note: Boldface text indicates a significant linear relationship.

Abbreviation: BMD, bone mineral density; CTX, carboxy terminal telopeptide of collagen type I; EBMIL, excess BMI loss; LSG, laparoscopic sleeve gastrectomy; OC, osteocalcin; VAT, visceral adipose tissue. artefact of reduced measurement sensitivity following changes in weight rather than actual physiological changes in bone tissue, ${ }^{30}$ the increases in bone formation and resorption marker levels in our study may suggest that the reduction in BMD was possibly a real effect. Moreover, our multiple linear regression results showed that the increases in the markers of bone formation and resorption are closely related to the reduction in BMD (Table 3). The above evidence suggests that increases in bone formation and resorption may play a role in the decrease in BMD after LSG in Chinese patients with obesity. Furthermore, researchers have found that peptide YY acts as a negative regulator of osteoblastic bone formation ${ }^{31}$ and that ghrelin directly stimulates bone formation. ${ }^{32}$ The peptide YY was increased after LSG, while ghrelin was decreased. ${ }^{33}$ Although our study did not detect gut hormones, the above evidence suggests that changes in gut hormones may be involved in the pathogenesis of bone loss following LSG.

Our study showed that the EBMIL and the decrease in VAT mass were correlated with the decreases in BMD in the femoral neck and total hip after LSG (Table 3). Similarly, bone loss has been demonstrated at various bone sites after nonsurgical weight loss. ${ }^{34}$ Our data suggest that one mechanism for bone loss following weight loss is a reduced mechanical load on the skeleton and a subsequent decrease in bone mass that results from weight loss. Our results also suggest that the reduction in VAT mass was negatively correlated with the decrease in BMD after LSG. It has been found that excess VAT may be deleterious to BMD. ${ }^{35}$ The potential mechanism may be associated with increased production of pro-inflammatory cytokines such as IL-6, ${ }^{36}$ which could stimulate bone resorption in osteoclasts. ${ }^{37}$ The evidence above indicates that a decrease in VAT mass may have a protective effect on the decrease in BMD in patients with obesity who undergo LSG. It is necessary to pay attention to the 
reductions in BMI and VAT mass after LSG, and physical exercises should be performed to prevent a decrease in BMD during weight loss. ${ }^{38,39}$

In our research, the levels of vitamin D before and after surgery were both deficient, while the levels of serum calcium, phosphorus and iPTH were in the normal ranges (Table 1). Although it was not clear whether LSG can cause malabsorption of vitamin $\mathrm{D}$, these data suggest that we should encourage supplementation with vitamin D before and after LSG. Vitamin D is essential for appropriate rates of calcium absorption and bone metabolism. A deficiency in vitamin $\mathrm{D}$ with a high prevalence of secondary hyperparathyroidism and high bone turnover was detected after metabolic surgery. ${ }^{40}$ It has been shown that ongoing vitamin D supplementation can decrease the rate of BMD loss after metabolic surgery, even though the increase in bone turnover markers is less pronounced. ${ }^{38}$ This evidence suggests that preventative measures for bone loss should be initiated in the first months after surgery, which is a period associated with increased bone turnover. In our study, all the participants were instructed to take vitamin D 800 IU, but only $44.9 \%$ of patients had supplemented. It has been recommended that LSG and RYGB patients take an initial dosage of at least 3000 IU vitamin D daily along with 1200 to $1500 \mathrm{mg}$ of calcium supplements. ${ }^{41}$ This result indicates that the dose of vitamin D in our study was not enough and that the compliance of patients was also important. Moreover, the time of sunlight exposure has a large impact on the level of vitamin D. ${ }^{42}$ Although no secondary hyperparathyroidism occurred in our study, the BMD of the population with obesity significantly decreased, and the bone formation and resorption marker levels increased. In the future, in addition to taking sufficient amounts of vitamin D orally, we also need to determine whether the sunlight exposure time is sufficient.

The present study was limited by the sample size and follow-up period, which may influence the results. Studies with larger sample sizes and longer follow-up times are warranted. Another limitation was that DXEA measured BMD. It has been found that increasing the thickness of overlying fat induces inaccuracies and measurement errors when BMD is assessed using DXEA. ${ }^{43}$ Although overlying fat also affects quantitative computed tomography (QCT) BMD measurements, the error is smaller and more uniform than with DXEA BMD. ${ }^{43,44}$ Caution must be used when interpreting DXA BMD results of clinical studies in which weight and body composition are changing.
In summary, our study showed reductions in BMD of the femoral neck and total hip in Chinese patients with obesity after weight loss due to LSG, while the bone formation and resorption marker levels increased. The significant reduction in BMD was related to the EBMIL, the reduction in VAT mass and the increases in bone formation and absorption marker levels. Whether the reduction in BMD is secondary to physiological adaptations of bone metabolism to reduced mechanical constraints or whether bone loss is pathological in itself with an increased fracture risk remains to be determined.

\section{Abbreviations}

BMD, bone mineral density; BMI, body mass index; CSU, Central South University; CTX, carboxy terminal telopeptide of collagen type I; DEXA, dual energy X-ray absorptiometry; EBMIL, excess BMI loss; FCP, fasting C-peptide; FPG, fasting plasma glucose; $\mathrm{HbA}_{1 \mathrm{c}}$, haemoglobin $\mathrm{A}_{1 \mathrm{c}}$; iPTH, intact parathyroid hormone; LSG, laparoscopic sleeve gastrectomy; OC, osteocalcin; QCT, quantitative computed tomography; RYGB, Roux-en-Y gastric bypass; TPINP, total-terminal propeptide of type I procollagen; VAT, visceral adipose tissue.

\section{Acknowledgment}

We thank Zhongjian Xie (Department of Metabolism and Endocrinology, Hunan Provincial Key Laboratory of Metabolic Bone Diseases and National Clinical Research Center for Metabolic Diseases, The Second Xiangya Hospital, Central South University, Changsha, Hunan, China) for critically reading the manuscript.

\section{Funding}

This study was supported by the National Key R\&D Program of China (2016YFC1305000, 2016YFC1305001), the National Natural Science Foundation of China (91749118, 81770775, 81670481), the Science and Technology Major Project of Hunan Province (2017SK1020) and the Planned Science and Technology Project of Hunan Province (2017RS3015).

\section{Disclosure}

The authors declare that they have no conflicts of interest.

\section{References}

1. NCD-RisC NRFC. Trends in adult body-mass index in 200 countries from 1975 to 2014: a pooled analysis of 1698 population-based measurement studies with $19 \cdot 2$ million participants. Lancet. 2016;10026 (387):1377-1396. 
2. Salminen P, Helmio M, Ovaska J, et al. Effect of laparoscopic sleeve gastrectomy vs laparoscopic roux-en-y gastric bypass on weight loss at 5 years among patients with morbid obesity: the SLEEVEPASS randomized clinical trial. JAMA. 2018;319(3):241-254. doi:10.1001/ jama.2017.20313

3. Rubino F, Nathan DM, Eckel RH, et al. Metabolic surgery in the treatment algorithm for type 2 diabetes: a joint statement by international diabetes organizations. Diabetes Care. 2016;39(6):861-877.

4. Khorgami Z, Shoar S, Andalib A, et al. Trends in utilization of bariatric surgery, 2010-2014: sleeve gastrectomy dominates. Surg Obes Relat Dis. 2017;13(5):774-778. doi:10.1016/j.soard.2017.01.031

5. Johansson H, Kanis JA, Oden A, et al. A meta-analysis of the association of fracture risk and body mass index in women. $J$ Bone Miner Res. 2014;29(1):223-233. doi:10.1002/jbmr.2017

6. Cosman F, de Beur SJ, LeBoff MS, et al. Clinician's guide to prevention and treatment of osteoporosis. Osteoporos Int. 2014;25 (10):2359-2381. doi:10.1007/s00198-014-2794-2

7. Evans AL, Paggiosi MA, Eastell R, Walsh JS. Bone density, microstructure and strength in obese and normal weight men and women in younger and older adulthood. $J$ Bone Miner Res. 2015;30 (5):920-928. doi:10.1002/jbmr.2407

8. Yang S, Shen X. Association and relative importance of multiple obesity measures with bone mineral density: the National Health and Nutrition Examination Survey 2005-2006. Arch Osteoporos. 2015;10:14. doi:10.1007/s11657-015-0219-2

9. Grethen E, Hill KM, Jones R, et al. Serum leptin, parathyroid hormone, 1,25-dihydroxy vitamin $\mathrm{D}$, fibroblast growth factor 23 , bone alkaline phosphatase, and sclerostin relationships in obesity. $J$ Clin Endocrinol Metab. 2012;5(97):1655-1662. doi:10.1210/jc.2011-2280

10. Hsu YH, Venners SA, Terwedow HA, et al. Relation of body composition, fat mass, and serum lipids to osteoporotic fractures and bone mineral density in Chinese men and women. Am J Clin Nutr. 2006;83 (1):146-154. doi:10.1093/ajcn/83.1.146

11. Viljakainen HT, Pekkinen M, Saarnio E, et al. Dual effect of adipose tissue on bone health during growth. Bone. 2011;48(2):212-217. doi:10.1016/j.bone.2010.09.022

12. Tanaka S, Kuroda T, Saito M, Shiraki M. Overweight/obesity and underweight are both risk factors for osteoporotic fractures at different sites in Japanese postmenopausal women. Osteoporos Int. 2013;24(1):69-76. doi:10.1007/s00198-012-2209-1

13. Ko BJ, Myung SK, Cho KH, et al. Relationship between bariatric surgery and bone mineral density: a meta-analysis. Obes Surg. 2016;26(7):1414-1421. doi:10.1007/s11695-015-1928-8

14. Adamczyk P, Buzga M, Holeczy P, et al. Bone mineral density and body composition after laparoscopic sleeve gastrectomy in men: a short-term longitudinal study. Int J Surg. 2015;23(Pt A):101-107. doi:10.1016/j.ijsu.2015.09.048

15. Ruiz-Tovar J, Oller I, Priego P, et al. Short- and mid-term changes in bone mineral density after laparoscopic sleeve gastrectomy. Obes Surg. 2013;23(7):861-866. doi:10.1007/s11695-013-0866-6

16. Luhrs AR, Davalos G, Lerebours R, et al. Determining changes in bone metabolism after bariatric surgery in postmenopausal women. Surg Endosc. 2020;34(4):1754-1760. doi:10.1007/s00464-01906922-8

17. Cadart O, Degrandi O, Barnetche T, et al. Long-term effects of Roux-en-Y gastric bypass and sleeve gastrectomy on bone mineral density: a 4-year longitudinal study. Obes Surg. 2020;30 (9):3317-3325. doi:10.1007/s11695-020-04568-3

18. Wang X, Li L, Zhu C, Gao J, Qu S. Alteration of bone mineral density differs between genders in obese subjects after laparoscopic sleeve gastrectomy: bone morphogenetic protein 4 may count. Obes Surg. 2018;28(10):3221-3226. doi:10.1007/s11695-018-3298-5

19. Vasikaran S, Eastell R, Bruyere O, et al. Markers of bone turnover for the prediction of fracture risk and monitoring of osteoporosis treatment: a need for international reference standards. Osteoporos Int. 2011;22(2):391-420. doi:10.1007/s00198-010-1501-1
20. Kanis JA, Melton LR, Christiansen C, Johnston CC, Khaltaev N. The diagnosis of osteoporosis. J Bone Miner Res. 1994;9(8):1137-1141.

21. Kolta S, Ravaud P, Fechtenbaum J, Dougados M, Roux C. Follow-up of individual patients on two DXA scanners of the same manufacturer. Osteoporos Int. 2000;11(8):709-713. doi:10.1007/s001980070070

22. Holick MF, Binkley NC, Bischoff-Ferrari HA, et al. Evaluation, treatment, and prevention of vitamin D deficiency: an Endocrine Society clinical practice guideline. J Clin Endocrinol Metab. 2011;96(7):1911-1930. doi:10.1210/jc.2011-0385

23. Carrasco F, Basfi-Fer K, Rojas P, et al. Changes in bone mineral density after sleeve gastrectomy or gastric bypass: relationships with variations in vitamin D, ghrelin, and adiponectin levels. Obes Surg. 2014;24(6):877-884. doi:10.1007/s11695-014-1179-0

24. Nakamura KM, Haglind EGC, Clowes JA, et al. Fracture risk following bariatric surgery: a population-based study. Osteoporos Int. 2014;25(1):151-158. doi:10.1007/s00198-013-2463-x

25. Ahlin S, Peltonen M, Sjoholm K, et al. Fracture risk after three bariatric surgery procedures in Swedish obese subjects: up to 26 years follow-up of a controlled intervention study. J Intern Med. 2020;287(5):546-557. doi:10.1111/joim. 13020

26. Javanainen M, Pekkarinen T, Mustonen H, Scheinin T, Leivonen M. Two-year nutrition data in terms of vitamin $\mathrm{D}$, vitamin $\mathrm{B} 12$, and albumin after bariatric surgery and long-term fracture data compared with conservatively treated obese patients: a retrospective cohort study. Obes Surg. 2018;28(9):2968-2975. doi:10.1007/s11695-018-3336-3

27. Paccou J, Martignene N, Lespessailles E, et al. Gastric bypass but not sleeve gastrectomy increases risk of major osteoporotic fracture: French population-based cohort study. J Bone Miner Res. 2020;35 (8):1415-1423. doi:10.1002/jbmr.4012

28. Bruno C, Fulford AD, Potts JR, et al. Serum markers of bone turnover are increased at six and 18 months after Roux-en-Y bariatric surgery: correlation with the reduction in leptin. $J$ Clin Endocrinol Metab. 2010;95(1):159-166. doi:10.1210/jc.2009-0265

29. Schollenberger AE, Heinze JM, Meile T, et al. Markers of bone metabolism in obese individuals undergoing laparoscopic sleeve gastrectomy. Obes Surg. 2015;25(8):1439-1445. doi:10.1007/ s11695-014-1509-2

30. Van Loan MD, Johnson HL, Barbieri TF. Effect of weight loss on bone mineral content and bone mineral density in obese women. $\mathrm{Am}$ J Clin Nutr. 1998;67(4):734-738. doi:10.1093/ajcn/67.4.734

31. Leitch VD, Brassill MJ, Rahman S, et al. PYY is a negative regulator of bone mass and strength. Bone. 2019;127:427-435. doi:10.1016/j. bone.2019.07.011

32. Fukushima N, Hanada R, Teranishi H, et al. Ghrelin directly regulates bone formation. J Bone Miner Res. 2005;20(5):790-798.

33. Tsoli M, Chronaiou A, Kehagias I, Kalfarentzos F, Alexandrides TK. Hormone changes and diabetes resolution after biliopancreatic diversion and laparoscopic sleeve gastrectomy: a comparative prospective study. Surg Obes Relat Dis. 2013;9(5):667-677. doi:10.1016/j. soard.2012.12.006

34. Ricci TA, Chowdhury HA, Heymsfield SB, et al. Calcium supplementation suppresses bone turnover during weight reduction in postmenopausal women. J Bone Miner Res. 1998;13(6):1045-1050. doi:10.1359/jbmr.1998.13.6.1045

35. Zhu K, Hunter M, James A, et al. Relationship between visceral adipose tissue and bone mineral density in Australian baby boomers. Osteoporos Int. 2020. doi:10.1007/s00198-020-05556-0

36. Pou KM, Massaro JM, Hoffmann U, et al. Visceral and subcutaneous adipose tissue volumes are cross-sectionally related to markers of inflammation and oxidative stress: the Framingham Heart Study. Circulation. 2007;116(11):1234-1241. doi:10.1161/CIRCULATIONAHA.107.7105 09

37. Moonga BS, Adebanjo OA, Wang HJ, et al. Differential effects of interleukin- 6 receptor activation on intracellular signaling and bone resorption by isolated rat osteoclasts. $J$ Endocrinol. 2002;173 (3):395-405. 
38. Muschitz C, Kocijan R, Haschka J, et al. The impact of vitamin D, calcium, protein supplementation, and physical exercise on bone metabolism after bariatric surgery: the BABS study. J Bone Miner Res. 2016;31(3):672-682. doi:10.1002/jbmr.2707

39. Murai IH, Roschel H, Dantas WS, et al. Exercise mitigates bone loss in women with severe obesity after Roux-en-Y gastric bypass: a randomized controlled trial. J Clin Endocrinol Metab. 2019;104 (10):4639-4650. doi:10.1210/jc.2019-00074

40. Costa TL, Paganotto M, Radominski RB, Kulak CM, Borba VC. Calcium metabolism, vitamin D and bone mineral density after bariatric surgery. Osteoporos Int. 2015;26(2):757-764. doi:10.1007/ s00198-014-2962-4

41. Mechanick JI, Youdim A, Jones DB, et al. Clinical practice guidelines for the perioperative nutritional, metabolic, and nonsurgical support of the bariatric surgery patient-2013 update: cosponsored by American Association of Clinical Endocrinologists, the Obesity Society, and American Society for Metabolic \& Bariatric Surgery. Surg Obes Relat Dis. 2013;9(2):159-191.
42. Farrar MD, Webb AR, Kift R, et al. Efficacy of a dose range of simulated sunlight exposures in raising vitamin $\mathrm{D}$ status in South Asian adults: implications for targeted guidance on sun exposure. Am J Clin Nutr. 2013;97(6):1210-1216. doi:10.3945/ajcn.112.052639

43. Yu EW, Thomas BJ, Brown JK, Finkelstein JS. Simulated increases in body fat and errors in bone mineral density measurements by DXA and QCT. J Bone Miner Res. 2012;27(1):119-124. doi:10.1002/ jbmr.506

44. Catano JS, Saldarriaga S, Chaput CD, Giambini H. Dual-energy estimates of volumetric bone mineral densities in the lumbar spine using quantitative computed tomography better correlate with fracture properties when compared to single-energy BMD outcomes. Bone. 2020;130:115100. doi:10.1016/j.bone.2019.115100

Diabetes, Metabolic Syndrome and Obesity: Targets and Therapy

\section{Publish your work in this journal}

Diabetes, Metabolic Syndrome and Obesity: Targets and Therapy is an international, peer-reviewed open-access journal committed to the rapid publication of the latest laboratory and clinical findings in the fields of diabetes, metabolic syndrome and obesity research. Original research, review, case reports, hypothesis formation, expert opinion and commentaries are all considered for publication. The manuscript management system is completely online and includes a very quick and fair peer-review system, which is all easy to use. Visit http://www.dovepress.com/testimonials.php to read real quotes from published authors. 